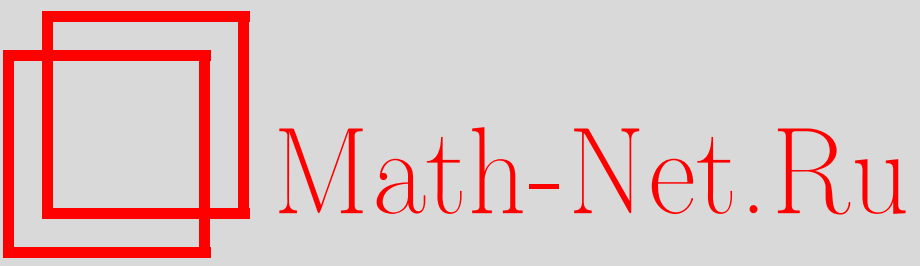

В. А. Попов, Алгебра Ли векторных полей Киллинга и ее стационарная подалгебра, Итоги науки и техн. Сер. Соврем. мат. и ее прил. Темат. обз., 2019, том 169, 56-66

DOI: https://doi.org/10.36535/0233-6723-2019-169-56-66

Использование Общероссийского математического портала Math-Net.Ru подразумевает, что вы прочитали и согласны с пользовательским соглашением

http://www. mathnet.ru/rus/agreement

Параметры загрузки :

IP : 54.81 .137 .203

26 апреля 2023 г., 14:01:29

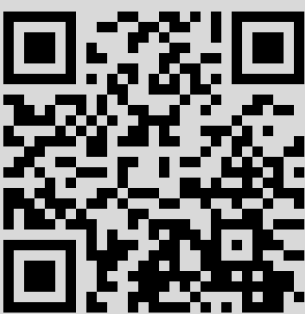




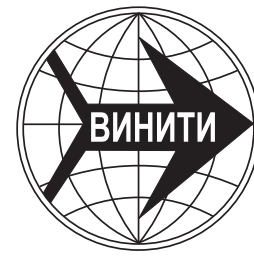

ИТОГИ НАУКИ И ТЕХНИКИ.

Современная математика и ее приложения.

Тематические обзоры.

Том 169 (2019). С. 56-66

DOI: 10.36535/0233-6723-2019-169-56-66

УДК 514.764

\title{
АЛГЕБРА ЛИ ВЕКТОРНЫХ ПОЛЕЙ КИЛЛИНГА И ЕЕ СТАЦИОНАРНАЯ ПОДАЛГЕБРА
}

\author{
(c) 2019 г. $\quad$ В. А. ПОПОВ
}

\begin{abstract}
АннотАция. Пусть $\mathfrak{g}$ - алгебра Ли всех векторных полей Киллинга на локально однородном римановом аналитическом многообразии $M, \mathfrak{h}$ - ее стационарная подалгебра, $G$ - односвязная группа, порожденная алгеброй $\mathfrak{g}, H$ - ее подгруппа, порожденная подалгеброй $\mathfrak{h}, \mathfrak{z}$ - центр алгебры $\mathfrak{g}$, $\mathfrak{r}$-ее радикал, а $[\mathfrak{g} ; \mathfrak{g}]$ - ее коммутант. Если $\operatorname{dim}(\mathfrak{h} \cap(\mathfrak{z}+[\mathfrak{g}, \mathfrak{g}]))=\operatorname{dim}(\mathfrak{h} \cap[\mathfrak{g}, \mathfrak{g}])$, то $H$ замкнута в $G$. Если для любой полупростой подалгебры $\mathfrak{p} \subset \mathfrak{g}$, удовлетворяющей условию $\mathfrak{p}+\mathfrak{r}=\mathfrak{g}$, имеет место равенство $(\mathfrak{p}+\mathfrak{z}) \cap \mathfrak{h}=\mathfrak{p} \cap \mathfrak{h}$, то $H$ замкнута в $G$. Изучено также аналитическое продолжение локально заданного риманова аналитического многообразия.
\end{abstract}

Ключевые слова: риманово многообразие, алгебра Ли, аналитическое продолжение, векторное поле, группа Ли, замкнутая подгруппа.

\section{LIE ALGEBRA OF KILLING VECTOR FIELDS AND ITS STATIONARY SUBALGEBRA}

\author{
(c) 2019 V. A. POPOV
}

\begin{abstract}
Let $\mathfrak{g}$ be the Lie algebra of all Killing vector fields on a locally homogeneous, analytic Riemannian manifold $M, \mathfrak{h}$ be a stationary subalgebra of $\mathfrak{g}, G$ be the simply connected group generated by the algebra $\mathfrak{g}, H$ be the subgroup of $G$ generated by the subalgebra $\mathfrak{h}, \mathfrak{z}$ be the center of the algebra $\mathfrak{g}$, $\mathfrak{r}$ be its radical, and $[\mathfrak{g} ; \mathfrak{g}]$ be its commutator subgroup. If $\operatorname{dim}(\mathfrak{h} \cap(\mathfrak{z}+[\mathfrak{g}, \mathfrak{g}]))=\operatorname{dim}(\mathfrak{h} \cap[\mathfrak{g}, \mathfrak{g}])$, then $H$ is closed in $G$. If for any semisimple subalgebra $\mathfrak{p} \subset \mathfrak{g}$ satisfying the condition $\mathfrak{p}+\mathfrak{r}=\mathfrak{g}$, the relation $(\mathfrak{p}+\mathfrak{z}) \cap \mathfrak{h}=\mathfrak{p} \cap \mathfrak{h}$ holds, then $H$ is closed in $G$. We also examine the analytic continuation of the given local, analytic Riemannian manifold.
\end{abstract}

Keywords and phrases: Riemannian manifold, Lie algebra, analytic continuation, vector field, Lie group, closed subgroup.

AMS Subject Classification: 53C20, 54H15

1. Введение. Однородное пространство определяется группой Ли $G$ и ее замкнутой подгруппой Ли $H$. Локально однородное риманово аналитическое многообразие определяется алгеброй $\mathfrak{g}$ Ли векторных полей Киллинга и ее стационарной подалгеброй $\mathfrak{h}$. Пусть $G$ - односвязная группа, порожденная алгеброй $\mathfrak{g}$ и $H$ - ее подгруппа, порожденная подалгеброй $\mathfrak{h}$. Если $G$ является алгеброй Ли всех векторных полей Киллинга локально заданной римановой аналитической метрики, то эта метрика аналитически продолжается до метрики однородного пространства тогда и только тогда, когда $H$ замкнута в $G$.

Строение незамкнутых подгрупп хорошо известно. Однако соответствующие исследования носят число алгебраический характер и никак не учитывают локальные свойства римановой метрики. Описание свойств незамкнутой подгруппы $H \subset G$ содержится в классической работе А. И. Мальцева [8]. Если подгруппа Ли $H$ односвязной группы Ли $G$ не замкнута в $G$, то группа $G$ содержит такой тор $T$, что пересечение $H \cap T$ является всюду плотной обмоткой этого тора. 
Однако этот факт трудно установить исходя из локальных свойств заданной римановой аналитической метрики, т.е. исходя из свойств алгебры Ли $\mathfrak{g}$ и стационарной подалгебры $\mathfrak{h}$. Можно ли найти свойства алгебры Ли всех векторных полей Киллинга, при выполнении которых подгруппа $H$, определяемая стационарной подалгеброй $\mathfrak{h}$, будет замкнутой в односвязной группе $G$, порожденной алгеброй $\mathfrak{g}$ ? В этом направлении отметим результат Мостова, согласно которому $H$ замкнута в $G$, если $\mathfrak{h}$ полупроста. Кроме того, Мостов доказал, что $H$ замкнута в $G$, если $\operatorname{dim} \mathfrak{g}-\operatorname{dim} \mathfrak{h}<5$ (см. [9]).

Можно ли найти необходимые и достаточные свойства алгебры Ли $\mathfrak{g}$ всех векторных полей Киллинга на римановом аналитическом многообразии $M$ и ее стационарной подалгебры $\mathfrak{h}$, при выполнении которых $H$ замкнута в $G$ ? Чисто алгебраических средств здесь недостаточно. Для исследования проблемы замкнутости стационарной подгруппы $H$ в односвязной группе $G$ привлекается изучение аналитического продолжения локально заданной римановой аналитической метрики. Многообразия, являющиеся аналитическим продолжением произвольной локально заданной римановой аналитической метрики, имеют одну и ту же алгебру Ли всех векторных полей Киллинга. Поэтому вопрос замкнутости группы $H$ в $G$ эквивалентен вопросу об аналитической продолжаемости данной локально заданной римановой аналитической метрики на локально однородном пространстве до метрики полного многообразия. Понятие аналитического продолжения римановой аналитической метрики присутствовало в классических монографиях Хелгасона [7] и Ш. Кобаяси и К. Номидзу [1], но развития не получило.

В работах $[4-6,10,11]$ изучается случай, когда $\mathfrak{g}$ имеет нулевой центр, причем не только для римановых многообразий, но также для псевдоримановых пространств и пространств аффинной связности. Доказано, что в этом случае подгруппа $H$, определяемая стационарной подалгеброй $\mathfrak{h}$, будет замкнутой в односвязной группе $G$, порожденной алгеброй $\mathfrak{g}$. Помимо алгебраического подхода, развивается аналитический подход для изучения аналитического продолжения римановых аналитических многообразий. Основной темой данной работы является исследование локально однородных многообразий, алгебра Ли g всех векторных полей Киллинга которых имеет нетривиальный центр $\mathfrak{z}$. Выводятся свойства алгебры $\mathfrak{g}$, ее стационарной подалгебры $\mathfrak{h}$ и центра $\mathfrak{z}$, обеспечивающего замкнутость подгруппы $H$, определяемой стационарной подалгеброй $\mathfrak{h}$, в односвязной группе $G$, порожденной алгеброй $\mathfrak{g}$. Пусть $\mathfrak{z}$ - центр алгебры $\mathfrak{g}, \mathfrak{r}$ - ее радикал, а $[\mathfrak{g} ; \mathfrak{g}]-$ ее коммутант. Если

$$
\operatorname{dim}(\mathfrak{h} \cap(\mathfrak{z}+[\mathfrak{g}, \mathfrak{g}]))=\operatorname{dim}(\mathfrak{h} \cap[\mathfrak{g}, \mathfrak{g}]),
$$

то $H$ замкнута в $G$. Если для любой полупростой подалгебы $\mathfrak{p} \subset \mathfrak{g}$, обладающей свойством $\mathfrak{p}+\mathfrak{r}=\mathfrak{g}$, где $\mathfrak{r}$ - радикал $\mathfrak{g}$, имеет место равенство $(\mathfrak{p}+\mathfrak{z}) \cap \mathfrak{h}=\mathfrak{p} \cap \mathfrak{h}$, то $H$ замкнута в $G$.

2. Аналитическое продолжение римановых многообразий. Рассмотрим риманово аналитическое многообразие $M$ и шар $U \subset M$ малого радиуса с центром в некоторой точке $x_{0} \in M$. Под аналитическим продолжением локально заданной метрики будем подразумевать любое риманово аналитическое многообразие $N$, для которого существует аналитическая изометрия $\varphi: U \rightarrow N$. Поставим задачу найти наиболее естественное аналитическое продолжение данной метрики. Естественным требованием является свойство непродолжаемости искомого многообразия, введенного еще в классических монографиях $[1,7]$. Однако непродолжаемые многообразия могут быть весьма своеобразными, например, односвязная накрывающая правой полуплоскости с выколотыми точками $(1 / n ; k / n), k, n \in \mathbb{N}$.

В исследованиях по геометрии римановых пространств в целом, как правило, существенным требованием является полнота рассматриваемого многообразия. Для полного односвязного риманова аналитического многообразия любая изометрия $\varphi: U \rightarrow V$ между двумя связными открытыми подмножествами $U \subset M, V \subset M$ аналитически продолжается до изометрии $\varphi: M \rightarrow M$ (см. [1]).

Однако в общем случае шар $U$ риманова аналитического многообразия нельзя изометрически вложить в полное риманово аналитическое многообразие, т.е., вообще говоря, локально заданная риманова метрика аналитически не продолжается до метрики полного риманова многообразия. 
Возникает вопрос об обобщении понятия полноты. Естественным обобщением такого рода является непродолжаемость риманова аналитического многообразия. Однако структура непродолжаемых многообразий может быть весьма причудливой.

Зададимся вопросом, можно ли по заданным локальным свойствам римановой аналитической метрики, т.е. метрики, заданной на малом шаре $U$, построить риманово аналитическое многообразие $M$, содержащее $U$ в качестве открытого подмножества и допускающее аналитическое продолжение локальных изометрий до изометрий всего многообразия ${ }^{1}$ ? Непреодолимым препятствием для такого продолжения является следующий факт. Пусть $\mathfrak{g}$ - алгебра Ли всех векторных полей Киллинга на римановом аналитическом многообразии $M$ и $\mathfrak{h}_{p} \subset \mathfrak{g}$ - ее стационарная подалгебра, для фиксированной точки $p \in M$

$$
X \in \mathfrak{h}_{p} \Longleftrightarrow X(p)=0 .
$$

Пусть $G$ - односвязная подгруппа, порожденная алгеброй $\mathfrak{g}$, и $H$-ее подгруппа, порожденная подалгеброй $\mathfrak{h}$. Пусть $G$ действует на односвязном многообразии $M$; тогда орбита фиксированной точки $p \in M$ является подмногообразием, изометричным фактор-группе $G / H$. Но фактор группа $G / H$ является многообразием лишь в случае замкнутости подгруппы $H$ в $G$, а это выполняется не всегда.

Перейдем к точным определениям и формулировкам.

Определение 1. Векторное поле $X=\xi^{i}(x)$ на римановом многообразии $M$ называется инфинитезимальной изометрией, или векторным полем Киллинга, если производная Ли метрического тензора $g_{i j}$ вдоль этого поля равна $0, \mathcal{L}_{X} g=0$. В локальных координатах

$$
\frac{\partial g_{i j}}{\partial x^{k}} \xi^{k}+g_{k j} \frac{\partial \xi^{k}}{\partial x^{i}}+g_{i k} \frac{\partial \xi^{k}}{\partial x^{j}}=0
$$

Определение 2. Локальной изометрией между двумя римановыми аналитическими многообразиями $M$ и $N$ называется изометрия $\varphi: U \rightarrow V$ между открытыми подмножествами $U \subset M$, $V \subset M$. Многообразия, между которыми существует локальная изометрия, назовем локалъно изометричными.

Любое векторное поле $X \in \mathfrak{g}$ аналитически продолжается вдоль любой кривой на многообразии $M$; тем самым, алгебра Ли $\mathfrak{g}$ определяет алгебру Ли $\mathfrak{g}$ векторных полей Киллинга на любом односвязном многообразии $N$, локально изометричном $M$. Этот факт верен также для многообразий аффинной связности.

Лемма 1. Пусть $M$-аналитическое многообразие аффинной связности, $X$ - инфинитезимальное афбинное преобразование, заданное в области $U \subset M$, и пусть $\gamma(t), 0 \leqslant t \leqslant 1,-$ такая непрерывная кривая в $M$, что $\gamma(0) \subset U$. Тогда векторное поле $X$ аналитически продолжсаемо вдоль $\gamma(t)$. Если кривые $\gamma(t)$ и $\delta(t), 0 \leqslant t \leqslant 1, \gamma(0)=\delta(0), \gamma(1)=\delta(1)=x_{1}$, гомотопны, то продолжения векторных полей в точку $x_{1}$ вдоль этих кривых совпадают.

Доказательство. Предположим, что векторное поле $X$ аналитически продолжаемо в окрестность любой точки $\gamma(t)$ при $0 \leqslant t \leqslant t_{1}$. Докажем, что $X$ продолжается и в окрестность точки $q=\gamma\left(t_{1}\right)$. Пусть $V$ - нормальная окрестность точки $q$, являющаяся нормальной окрестностью каждой из своих точек (см. [8]). Рассмотрим такое $t \leqslant t_{1}$, что $p=\gamma(t) \in V$.

Векторное поле $X$ порождает локальную однопараметрическую группу изометрий $\phi_{s}$ в окрестности каждой точки $\gamma(t), t \leqslant t_{1}$. Докажем, что для всех достаточно малых значений $s$ локальные изометрии $\phi_{s}$ аналитически продолжаются и в окрестность точки $q=\gamma\left(t_{1}\right)$. Тогда векторное поле скоростей этой локальной группы изометрий и будет аналитическим продолжением векторного поля $X$ в окрестность точки $q$.

Рассмотрим связное открытое множество $V_{0} \subset V$, содержащее точки $p$ и $q$, замыкание которого также принадлежит $V, \overline{V_{0}} \subset V, p, q \in V_{0}$. Рассмотрим малую окрестность $V^{\prime} \subset V_{0}$ точки $q$ и соединим точку $p$ отрезком геодезической $\alpha(t), 0 \leqslant t \leqslant 1$, с произвольной точкой $q^{\prime} \in V^{\prime}$. Пусть

\footnotetext{
${ }^{1}$ Это означает, что любая изометрия $\varphi: U \rightarrow V$ между двумя связными открытыми подмножествами $U \subset M$, $V \subset M$ аналитически продолжается до изометрии $\varphi: M \rightarrow M$.
} 
$Y=\frac{d \alpha}{d t}(0) \in T_{p} M$ и $p_{s}=\varphi_{s}(p), Y_{s}=\varphi_{s}(Y)$. Из точки $p_{s}$ проведем такую геодезическую $\beta(t)$, $0 \leqslant t \leqslant 1$, что $\frac{d \beta}{d t}=Y_{s}$. При достаточно малых значениях $s$ имеем $\beta(t) \in V_{0}, 0 \leqslant t \leqslant 1$. Положим $\varphi_{s}\left(q^{\prime}\right)=\beta(1)$. Полученное таким образом отображение и есть аналитическое продолжение аффинного преобразования $\varphi_{s}$.

Принципиальным является исследование случая вполне неоднородной римановой метрики, т.е., метрики, не допускающей никаких движений (полей Киллинга). В этом случае удается определить так называемое квазиполное многообразие, обладающее свойством непродолжаемости и единственности для каждой локально заданной вполне неоднородной метрики (см. [2]).

Определение 3. Риманово аналитическое многообразие называется вполне неоднородным многообразием, если на нем не существует векторных полей Киллинга. Риманову метрику вполне неоднородного многообразия назовем вполне неоднородной метрикой.

Согласно лемме 1 все многообразия, локально изометричные вполне неоднородному многообразию, являются вполне неоднородными.

Определение 4. Вполне неоднородное ориентированное риманово аналитическое многообразие называется квазиполным, если оно непродолжаемо и не допускает нетривиальных сохраняющих ориентацию локальных изометрий в себя.

Приведем основные свойства вполне неоднородных и квазиполных многообразий (см. [2]). Для произвольного вполне неоднородного многообразия $M$ рассмотрим множество $S \subset M$ всех неподвижных точек всевозможных сохраняющих ориентацию локальных изометрий многообразия $M$ в себя.

Теорема 1. Для произвольного вполне неоднородного риманова аналитического многообразия $M^{\prime}$ множество $S \subset M^{\prime}$ является аналитическим подмножеством коразмерности $\geqslant 2$. Следовательно, $M^{\prime} \backslash S$ является связным многообразием.

Теорема 2. Для любого вполне неоднородного риманова аналитического многообразия $M^{\prime}$ существует локально изометричное ему квазиполное многообразие $M$ и локально изометричекое накрывающее отображсение $f: M^{\prime} \backslash S \rightarrow M$. Таким образом, квазиполное многообразие обладает свойством единственности для каждой вполне неоднородной локально заданной римановой аналитической метрики.

Определение квазиполного многообразия удается обобщить на случай, когда алгебра Ли всех векторных полей Киллинга для заданной локально определенной римановой аналитической метрики не имеет центра (см. [3]).

Определение 5. Вполне неоднородное ориентированное риманово аналитическое многообразие, алгебра векторных полей которого имеет нулевой центр, называется квазиполным, если оно непродолжаемо и не допускает нетривиальных локальных изометрий в себя, сохраняющих ориентацию и все векторные поля Киллинга.

Определение 6. Риманово аналитическое многообразие $M$ называется локально однородным, если в любой точке $p \in M$ векторные поля Киллинга образуют базис касательного пространства $T_{p} M$.

Эквивалентное определение локально однородного многообразия $M$ состоит в том, что любых точек $p, q \in M$ существует такая локальная изометрия $\varphi$ многообразия $M$, что $\varphi(p)=q$.

Исследуем ориентированные римановы аналитические многообразия, алгебра Ли всех векторных полей Киллинга которых не имеет центра. Наша цель - доказать, что каждое такое многообразие локально изометрично квазиполному многообразию, а локально однородное квазиполное многообразие является полным однородным многообразием.

Обозначим через $Z(M)$ псевдогруппу всех сохраняющих ориентацию локальных изометрий риманова аналитического многообразия $M, \varphi \in Z(M) \Longleftrightarrow X \in \mathfrak{g}$. 
Лемма 2. Пусть $M$-риманово аналитическое многообразие, удовлетворяющее свойству однозначного продолжения векторных полей Киллинга, причем алгебра Ли всех векторных полей Киллинга на $M$ не имеет иентра. Тогда множество $S \subset M$, состоящее из неподвижсных точек всевозможных изометрий $\varphi \in Z(M)$, является аналитическим подмножеством коразмерности $\geqslant 2$.

Доказательство. Докажем, что для любого открытого множества $U \subset M$ с компактным замыканием имеется только конечное число локальных изометрий из $U$ в себя, принадлежащих псевдогруппе $Z(M)$. Предположим противное и рассмотрим бесконечную последовательность локальных изометрий $\varphi_{i} \in Z(M)$, область определения и множество значений которых лежат в $U$. При доказательстве леммы 3 работы [2] по бесконечной последовательности локальных изометрий $\varphi_{i}$ на некотором открытом множестве $V \subset U$ было построено векторное поле Киллинга $X$, которое при переходе к подпоследовательности удовлетворяет следующему условию: для любого $t$, лежащего в промежутке $[-1 ; 1]$, существует такое $k(i) \in \mathbb{N}$, что $\lim _{i \rightarrow \infty} \varphi_{i}^{k(i)}=\operatorname{Exp} t X$, где $\operatorname{Exp} t X$ - локальная однопараметрическая группа изометрий, порожденная векторным полем $X$. Следовательно для любого векторного поля $Y$ на $V$ существует такое $\exists i \in \mathbb{N}$, что выполняется неравенство

$$
\begin{aligned}
& \left|(\operatorname{Exp} t x)_{*} Y-Y\right| \leqslant\left|\varphi_{i}^{k(i)} Y-Y\right|+\left|(\operatorname{Exp} t X)_{*} Y-\varphi_{i}^{k(i)} Y\right| \leqslant \\
& \leqslant 0+\left|Y-(\operatorname{Exp}(-t X))_{*} \varphi_{i}^{k(i)} Y\right| \leqslant \frac{1}{2}\left|(\operatorname{Exp} t x)_{*} Y-Y\right| .
\end{aligned}
$$

Следовательно, для всех $Y \in \mathfrak{g}$ имеем $(\operatorname{Exp} t X)_{*} Y=Y$, т.е. $[X, Y]=0$. Это противоречит отсутствию центра в алгебре $\mathfrak{g}$.

Полученное противоречие доказывает существование только конечного числа локальных изометрий из $U$ в $U$, принадлежащих псевдогруппе $Z(M)$. Отсюда, как было показано в [2], уже легко следует тот факт, что множество $S$ является аналитическим подмножеством коразмерности $\geqslant 2$.

В силу леммы 2 многообразие $M \backslash S$ связно.

Лемма 3. Пусть $M$-риманово аналитическое многообразие, удовлетворяющее свойству однозначного продолжения векторных полей Киллинга, у которого и алгебра Ли всех векторных полей Киллинга не имеет чентра. Тогда существует локально изометрическое накрывающее отображение из $M \backslash S$ в риманово аналитическое многообразие $M_{1}$, также удовлетворлющее свойству однозначного продолжения векторных полей Киллинга, псевдогруппа $Z\left(M_{1}\right)$ которого состоит только из тождественного преобразования.

Доказательство. Факторизуем многообразие $M^{\prime} \backslash S$ по псевдогруппе $Z(M)$. Из доказательства леммы 2 следует, что для каждой точки $x \subset M \backslash S$ существует окрестность $U_{1 x} \in M \backslash S$ точки $x$, которая не допускает нетождественных сохраняющих ориентацию локальных изометрий в себя, принадлежащих псевдогруппе $Z(M)$. Это доказывает, что фактор-отображение $\pi$, проектирующее многообразие $M \backslash S$ во множество $M_{1}=M \backslash S / Z(M)$, является накрывающим отображением. Значит, для каждой точки $x \in M$ существует такая ее окрестность $U_{x} \subset M_{1}$ и такое открытое множество $V_{x} \subset \pi^{-1}\left(U_{x}\right)$, что отображение $\pi$ устанавливает гомеоморфизм между множествами $V_{x}$ и $U_{x}$. Определим риманово скалярное произведение. Сузив, если необходимо, множество $V_{x} \subset M \backslash S$, будем считать, что $V_{x}$ является координатной окрестностью точки $y \in \pi^{-1}\left(U_{x}\right) \subset M \backslash S$. Тогда объявим множество $U_{x} \subset M_{1}$ координатной окрестностью точки $x \in M_{1}$. Рассмотрим две такие окрестности $U_{1}, U_{2} \subset M_{1}, U_{1} \cap U_{2} \neq 0$. Заметим, что соответствующие множествам $U_{1}, U_{2}$ множества $V_{1}, V_{2} \subset M \backslash S$ могут и не пересекаться. Положим

$$
\pi^{-1}\left(U_{1} \cap U_{2}\right) \cap V_{1}=V_{10}, \quad \pi^{-1}\left(U_{1} \cap U_{2}\right) \cap V_{2}=V_{12} .
$$

Тогда существует изометрия $\alpha: V_{10} \simeq V_{20}$. Пусть $\psi_{1}$ и $\psi_{2}$ - координатные отображения на $V_{1}$ и $V_{2}$ соответственно. Тогда $\psi_{1} \pi^{-1}$ и $\psi_{2} \pi^{-1}$ будут координатными отображениями на $U_{1}$ и $U_{2}$. 
Рассмотрим произвольную точку $x \in M_{1}$ и произвольные векторы $X, Y \in T_{x} M_{1}$. Рассмотрим какую-нибудь точку $\pi^{-1}(x) \subset M \backslash S$ и такие векторы $X_{1}, Y_{1} \in T_{y} M$, что $\pi_{*} X_{1}=X, \pi_{*} Y_{1}=Y$. Определим риманово скалярное произведение $\langle X ; Y\rangle$ равным имеющемуся на $T_{x} M$ риманову скалярному произведению $\left\langle X_{1} ; Y_{1}\right\rangle$. Если взять другую точку $z \in \pi^{-1}(x)$ и такие векторы $X_{2}, Y_{2} \in$ $T_{z} M$, что $\pi_{*} X_{2}=X, \pi_{*} Y_{2}$, то существует такая локальная изометрия $\varphi \in Z(M)$, что $\varphi(z)=$ $y, \varphi_{*} X_{2}=X_{1}, \varphi_{*} Y_{2}=Y_{1}$. Следовательно, $\left\langle X_{1} ; Y_{1}\right\rangle=\left\langle X_{2} ; Y_{2}\right\rangle$. Это доказывает корректность определения римановой метрики на $M_{1}$.

Построенное риманово многообразие $M_{1}$ не допускает нетождественных сохраняющих ориентацию локальных изометрий, индуцирующих тождественное преобразование на алгебре векторных полей Киллинга $\mathfrak{g}$. Проекция $\pi: M \backslash S \rightarrow M_{1}$ является локально изометрическим накрывающим отображением. Остается доказать свойство однозначного продолжения векторных полей Киллинга на $M_{1}$. Рассмотрим векторное поле Киллинга $X$, заданное на некотором открытом множестве $U \subset M_{1}$, и такие открытые множества $U_{0} \subset U$ и $V_{0} \subset M \backslash S$, что накрывающее отображение $\pi$ устанавливает изометрию между множествами $V_{0}$ и $U_{0}$. Тогда векторное поле $\pi_{*}^{-1} X$ однозначно продолжается с множества $V_{0} \subset M$ на все многообразие $M$ и задает векторное поле $Y$ на $M$. Пусть точки $y, z \in M \backslash S$ таковы, что $\pi(x)=\pi(y)$ и $\pi_{*} Y(z)=\pi_{*} \varphi_{*} Y(y)$. Так как $\pi \varphi=\pi$ по определению $\pi$, то $\pi_{*} \varphi_{*}=\pi_{*}$. Следовательно, $\pi_{*} Y(z)=\pi_{*} \varphi_{*} Y(y)=\pi_{*} Y(y)$. Это доказывает, что отображение $\pi$ однозначно проектирует векторное поле $Y$, заданное на $M$, на векторное поле $\pi_{*} Y$, заданное на многообразии $M_{1}$. Полученное векторное поле $\pi_{*} Y$ и будет аналитическим продолжением векторного поля $X$ на все многообразие $M_{1}$.

Теорема 3. Произволъное риманово аналитическое многообразие $M$, у которого алгебра Ли векторных полей Киллинга не имеет иентра, локально изометрично квазиполному многообразию.

Доказательство. Рассмотрим произвольное риманово аналитическое многообразие $M^{\prime}$, у которого алгебра Ли векторных полей Киллинга не имеет центра. Многобразие $M_{1}$, построенное при доказательстве леммы 3, не допускает локальных изометрий в себя, сохраняющих ориентацию и векторные поля Киллинга. Тогда квазиполным многообразием $M$ будет некоторое максимальное аналитическое продолжение многообразия $M_{1}$. Будем считать, что все многообразия, которые мы будем рассматривать при доказательстве теоремы, обладают свойством однозначного аналитического продолжения векторных полей Киллинга, т.е. алгебра Ли всех векторных полей Киллинга одинакова для всех многообразий и равна $\mathfrak{g}$. Если $M^{\prime}$ обладает этим свойством, то и многообразие $M_{1}$ им обладает.

Рассмотрим множество $\Lambda$, состоящее из аналитических продолжений $M_{\alpha}$ многообразия $M_{1}$, обладающих свойством однозначного продолжения векторных полей Киллинга и не допускающих локальных изометрий, тождественных на алгебре всех векторных полей Киллинга. Снабдим многообразие $M_{1}$ отмеченной точкой и отмеченным репером в отмеченной точке, а образы этой точки и этого репера отметим в многообразиях $M_{\alpha} \in \Lambda$. Введем на этом множестве следующее отношение порядка: $M_{\alpha} \leqslant M_{\beta}$, если существует изометрическое вложение $i_{\alpha \beta}: M_{\alpha} \rightarrow M_{\beta}$, переводящее отмеченную точку в отмеченную и отмеченный репер в отмеченный. В результате $\Lambda$ становится частично упорядоченным множеством. Рассмотрим произвольное линейно упорядоченное подмножество $\Delta$ множества $\Lambda$ и построим прямой предел семейства многообразий $M_{\alpha} \in \Delta$ и отображений $i_{\alpha \beta}$. Получим многообразие $M_{0}$, обладающее следующими свойствами. Для любого многообразия $M_{\alpha} \in \Delta$ существует изометрическое вложение $i_{\alpha}: M_{\alpha} \rightarrow M_{0}$, причем $i_{\alpha}\left(M_{\alpha}\right) \subset i_{\beta}\left(M_{\beta}\right)$, если $M_{\alpha} \leqslant M_{\beta}$, и $M_{0}=\bigcup M_{\alpha}$. Докажем, что $M_{\alpha} \in \Lambda$. Произвольное векторное поле $X$ на многообразии $M_{1}$ при помощи вложений $i_{1 \alpha}: M_{1} \rightarrow M_{\alpha}$ и $i_{\alpha}: M_{\alpha} \rightarrow M_{0}$ переносится на многообразие $i_{\alpha}\left(M_{\alpha}\right) \subset M_{0}$, причем $\left(i_{\alpha} i_{1 \alpha}\right)_{*} X=\left(i_{\beta} i_{1 \beta}\right)_{*} X$ на $i_{\alpha}\left(M_{\alpha}\right) \cap i_{\beta}\left(M_{\beta}\right)$, а векторное поле Киллинга $\left(i_{\alpha} i_{1 \alpha}\right)_{*} X$ однозначно продолжается с подмногообразия $i_{\alpha}\left(M_{\alpha}\right) \subset M_{0}$ на любое подмногообразие $i_{\beta}\left(M_{\beta}\right) \subset M_{0}, M_{\beta} \geqslant M_{\alpha}$, и значит, на все многообразие $M_{0}$. Таким образом, векторное поле Киллинга, заданное на произвольно малом открытом множестве $U \subset M_{0}$, однозначно продолжается до векторного поля Киллинга на $M_{0}$. 
Рассмотрим теперь локальную изометрию $\varphi \in Z(M)$. Пусть точка $x_{0} \in M_{0}$ принадлежит области определения изометрии $\varphi$. Тогда точки $x_{0}$ и $\varphi\left(x_{0}\right)$ лежат в некотором подмногообразии $i_{\alpha}\left(M_{\alpha} \subset M_{0}\right.$. Следовательно, $\varphi \in Z\left(i_{\alpha}\left(M_{\alpha}\right)\right)$, и поэтому $\varphi$ является тождественным преобразованием. Значит, псевдогруппа $Z\left(M_{0}\right)$ состоит только из тождественного преобразования. Итак, для произвольного линейно упорядоченного подмножества $\Delta \subset \Lambda$ мы построили верхнюю грань. По лемме Цорна множество $\Lambda$ имеет максимальный элемент. Мы утверждаем, что многообразие $M$, являющееся таким максимальным элементом, и будет искомым квазиполным многообразием. Требуется доказать, что $M$ непродолжаемо.

Предполагая противное, обозначим через $N$ нетривиальное продолжение многообразия $M$. Пусть, как и выше, $S \subset N$ обозначает множество неподвижных точек всевозможных локальных изометрий из псевдогруппы $Z(N)$. Точно так же, как и при доказательстве леммы 3 было факторизовано многообразие $M \backslash S$, факторизуем многообразие $N \backslash S$. В результате получим многообразие $L$, удовлетворяющее свойству однозначного продолжения векторных полей Киллинга и не допускающее локальных изометрий, сохраняющих ориентацию и все векторные поля Киллинга. Обозначим через $i$ вложение $i: M \rightarrow N$. Докажем, что $i(M) \cap S=\varnothing$. Если $x \in i(M)$, то и некоторый нормальный шар $B$ с центром в $x$ принадлежит $i(M)$. Если, кроме того, $x \in S$, то существует локальная изометрия $\varphi \in Z(M)$, удовлетворяющая условию $\varphi(x)=X$. Эта изометрия определяет изометрию шара $B$ в себя, заданную в нормальных координатах линейным отображением - дифференциалом изометрии $\varphi$. Но существование такой изометрии противоречит тривиальности псевдогруппы $Z(M)$. Таким образом, $i$ дает вложение $i: M \rightarrow N \backslash S$. Сквозное отображение $\pi i: M \rightarrow L$, где $\pi: N \backslash S \rightarrow L$ - построенное при доказательстве леммы 3 накрывающее отображение, является также вложением. Так как, если $\pi i(x)=\pi i(y)$, то существует такая локальная изометрия $\varphi \in Z(M)$, что $\varphi(x)=\varphi(y)$, следовательно $x=y$. В силу того, что $M-$ максимальный элемент множества $\Lambda, \pi i$ является изометрией, и $N \backslash S$ накрывает $M$.

Имеем накрывающее отображение $\pi: N \backslash S \rightarrow M$ и вложение $i: M \rightarrow N \backslash S$, причем $i(M)$ открыто в $N \backslash S$. Пусть имеется последовательность точек $x_{n} \in i(M)$, сходящаяся к $x \in N \backslash S$. Тогда последовательность $y_{n}=\pi\left(x_{n}\right)$ также сходится к некоторой точке $y \in M$. Но тогда, так как $x_{n}=i\left(y_{n}\right)$, то $x=i(y) \in M$. Это доказывает замкнутость $i(M)$ в $N \backslash S$. Итак, $N \backslash S$ несвязно или $N \backslash S=N$, Но несвязность $N \backslash S$ противоречит лемме 2. Поэтому $N \backslash S=M$. Докажем, что $S=\varnothing$. Предположим противное и рассмотрим нормальный шар $B$ с центром в некоторой точке $x \in S \subset N$. Существует изометрия шара $B$ в себя. Эта изометрия не оставляет неподвижными точки из $B \backslash S$ и поэтому является нетождественной локальной изометрией из псевдогруппы $Z(N \backslash S)$. Так как $N \backslash S=M$, то это противоречит тривиальности псевдогруппы $Z(M)$. Это доказывает, что $S=\varnothing, N=M$ и $M$ непролжаемо.

Теорема 4. Пусть $\varphi$-локальная изометрия из псевдополного многообразия $M$ в псевдополное многообразие $N$. Тогда $\varphi$ продолжается до изометрии $\varphi: M \simeq N$.

Доказательство. Рассмотрим произвольную точку $X \in M$ и гладкую кривую $\gamma(t), 0 \leqslant t \leqslant 1$, $\gamma(t) \in D(\varphi) \subset M, \gamma(1)=x \mathrm{x}$. Докажем, что изометрию $\varphi$, заданную в окрестности $U=D(\varphi) \subset M$ точки $x_{0}=\gamma(0)$, можно продолжить вдоль кривой $\gamma$. Предположим, что такого продолжения не существует. Рассмотрим минимальное число $t_{1} \in[0 ; 1]$ среди таких чисел $t$, что изометрия $\varphi$ не продолжается в окрестность точки $\gamma(t)$ вдоль кривой $\gamma$. Докажем, тем не менее, что вопреки предположению продолжение $\varphi$ на некоторую окрестность точки $\gamma\left(t_{1}\right)$ вдоль кривой $\gamma$ существует.

В силу предположения, сделанного относительно $t_{1}$, для всех $t \in\left[0 ; t_{1}\right)$ изометрия $\varphi$ определена в некоторой окрестности точки $\gamma(t)$, так что на $N$ определена кривая $\delta(t)=\varphi(\gamma(t)), 0 \leqslant t \leqslant t_{1}$. Пусть $x_{1}=\gamma\left(t_{1}\right)$ и $\varepsilon>0$ таково, что окрестность $U_{\varepsilon}=\left\{x \in M, \rho\left(x ; x_{1}\right) \leqslant \varepsilon\right\}$ является нормальной окрестностью каждой из своих точек. Так как для любого $y \in N$ и любого $\varepsilon_{0}>0$ существует такое $\alpha>0$, что для всех $t^{\prime} \in\left[0 ; t_{1}\right)$ и $t^{\prime \prime} \in\left[0 ; t_{1}\right)$, удовлетворяющих условиям $\left|t_{1}-t^{\prime}\right|<\alpha$, $\left|t_{1}-t^{\prime \prime}\right|<\alpha$, выполняются неравенства

$$
\left|\rho\left(y ; \delta\left(t^{\prime}\right)\right)-\rho\left(y, \delta\left(t^{\prime \prime}\right)\right)\right| \leqslant \rho\left(\delta\left(t^{\prime}\right) ; \delta\left(t^{\prime \prime}\right)\right) \leqslant \int_{t^{\prime}}^{t^{\prime \prime}} \sqrt{\left\langle\delta^{\prime}(t) ; \delta^{\prime}(t)\right\rangle} d t=\int_{t^{\prime}}^{t^{\prime \prime}}\left\langle\gamma^{\prime}(t) ; \gamma^{\prime \prime}(t)\right\rangle d t<\varepsilon_{0} .
$$


Следовательно, для любого $y \in N$ существует предел $\lim _{t \rightarrow t_{1}} \rho(y, \delta(t))=\rho_{1}(y)$. Рассмотрим множество $V_{\varepsilon}=\left\{y \in N \mid \rho_{1}(y)<\varepsilon\right\}$. Существует изометрия $\varphi=\psi^{-1}$ некоторой окрестности $V_{D} \subset V_{\varepsilon}$ множества $D=\left\{y \in N \mid y=\delta(t), t_{2} \leqslant t<t_{1}\right\}$ на окрестность $U_{D} \subset U_{\varepsilon}$ множества $B=\left\{x \in M \mid x=\gamma(t), t_{2} \leqslant t<t_{1}\right\}$. Докажем, что $\psi$ можно продолжить до изометрии $\psi: V \simeq V$. Докажем сначала, что $\psi$ можно продолжить вдоль любой кривой $\nu(s), 0 \leqslant s \leqslant 1$, на $V_{\varepsilon}$, где $\nu(0) \in V_{D}, \nu(1)=y$ - произвольная точка на $V_{\varepsilon}$. Если предположить, что это не так, то существует минимальное число $s_{1}$ среди чисел $u \in[0 ; 1]$, обладающих следующим свойством: $\psi$ не продолжается вдоль кривой $\nu(s)$ в какую-либо окрестность точки $\nu(u)$. Пусть $\sigma>0$ и $s_{2}<s_{1}$ таковы, что множество $B_{\sigma}=\left\{y \in N \mid \rho\left(y ; \nu\left(s_{2}\right)\right)<\sigma\right\}$ является нормальной окрестностью точки $\nu\left(s_{2}\right)$ и $\rho\left(\nu\left(s_{2} ; \nu\left(s_{1}\right)\right)<\sigma / 2\right.$. Следовательно, $\nu\left(s_{1}\right) \in B_{\sigma}$. Используя линейность отображения $\psi$ в нормальных координатах, можно продолжить изометрию $\psi$, определенную на некоторой окрестности точки $\nu\left(s_{2}\right)$, до изометрии $\psi$, определенной на всем множестве $B_{\sigma}$, которое является окрестностью точки $\nu(s)$. Это опровергает предположение о непродолжаемости $\psi$ вдоль кривой $\nu(s)$.

Докажем теперь, что продолжение изометрии $\psi$ вдоль всевозможных кривых на $V_{\varepsilon}$ дает однозначное отображение $\psi: V_{\varepsilon} \rightarrow U_{\varepsilon}$. Предположим противное. Тогда существует такая замкнутая жорданова кривая $\nu(t), 0 \leqslant t \leqslant 1, \nu(0)=\nu(1)$, на $V_{\varepsilon}$, что кривая $\beta(t)=\psi(\nu(t))$ на $U_{\varepsilon}$ будет незамкнутой, $\beta(0) \neq \beta(1)$. Но так как всевозможные аналитические продолжения изометрии $\psi$ индуцируют одинаковые отображения на алгебре векторных полей Киллинга, то изометрия вида $\psi \psi^{-1}$, переводящая $\beta(0)$ в $\beta(1)$, принадлежит псевдогруппе $Z(M)$, а это противоречит тому, что $M$ является квазиполным многообразием. Аналогично доказывается, что продолжение локальной изометрии $\phi=\psi^{-1}$ из $U_{\varepsilon}$ в $V_{\varepsilon}$ задает однозначное отображение на множестве $\varphi\left(V_{\varepsilon}\right) \subset U_{\varepsilon}$. Итак, имеем изометрическое вложение $V_{\varepsilon} \rightarrow U_{\varepsilon}$. Докажем, что оно является сюръективным отображением. Если предположить противное, то склеив многообразия $N$ и $U_{\varepsilon}$ по отображению $\psi$, получим нетривиальное продолжение многообразия $N$, что противоречит его непродолжаемости. Следовательно, имеем изометрию $\psi: V_{\varepsilon} \rightarrow U_{\varepsilon}$. Обратная изометрия $\psi^{-1}: U_{\varepsilon} \rightarrow V_{\varepsilon}$ дает продолжение изометрии $\psi$ на окрестность $U_{\varepsilon}$ точки $\gamma\left(t_{1}\right)$ вдоль кривой $\gamma$ вопреки первоначальному предположению относительно $t_{1}$.

Таким образом, мы доказали, что локальная изометрия $\varphi$ из $M$ в $N$ продолжается в любую точку $x \in M$ вдоль произвольной кривой на $M$. Точно так же, как выше мы доказали, что продолжение изометрии $\psi$ вдоль всевозможных кривых на $V_{\varepsilon}$ дает взаимно однозначное отображение, определенное на всем $V_{\varepsilon}$, можно доказать, что продолжение $\varphi$ вдоль всевозможных кривых на $M$ дает изометрическое вложение $\varphi: M \rightarrow N$.

Следствие 1. Произвольное риманово аналитическое многообразие, алгебра Ли векторных полей Киллинга которого не имеет центра, локально изометрично единственному квазиполному многообразию. Иными словами, локально заданная риманова аналитическая метрика, алгебра Ли векторных полей Киллинга которой не имеет центра, единственным образом продолжается до квазиполного многообразия.

Доказательство. Пусть квазиполное многообразие $M$ локально изометрично многообразию $M^{\prime}$ и пусть $N$ - другое квазиполное многообразие, локально изометричное многообразию $M^{\prime}$. Тогда существует локальная изометрия $\varphi$ из $N$ в $M^{\prime}$ и локальная изометрия $\psi$ из $M^{\prime}$ в $M$. Суперпозиция изометрий $\varphi$ и $\psi$ является локальной изометрией из $N$ в $M$. По теореме 4 локальная изометрия $\psi \varphi$ продолжается до изометрии $M \simeq N$. Что и требовалось доказать.

Следствие 2. Пусть $\mathfrak{g}$ - алгебра Ли всех векторных полей Киллинга на римановом аналитическом многообразии $M^{\prime}$, дифбеоморфном шару, а $\mathfrak{h}-$ ее стационарная подалгебра. Пусть $G-$ односвязная группа, порожденная алгеброй $\mathfrak{g}, u \mathrm{H}-$ ее подгруппа, порожденная подалгеброй $\mathfrak{h}$. Если $\mathfrak{g}$ не имеет центра, то $H$ замкнута в $G$.

Доказательство. Так как $M^{\prime}$ диффеоморфно шару, его векторные поля Киллинга аналитически продолжаются на нем однозначно. Согласно теореме 3 многообразие $M^{\prime}$ локально изоморфно квазиполному многообразию $M$, имеющему ту же самую алгебру Ли $\mathfrak{g}$ всех векторных полей 
Киллинга и ту же самую стационарную подалгебру $\mathfrak{h}$. Для произвольного векторного поля $X \in \mathfrak{g}$ при всех значениях параметра $t$, меньших некоторого числа $\delta$, элементы однопараметрической группы преобразований $\operatorname{Exp} t X$ являются локальными изометриями многообразия $M$. Согласно теореме 4 они продолжаются до изометрий всего многообразия $M$. Но тогда определены изометрии $\operatorname{Exp} n t X=(\operatorname{Exp} t X)^{n}$. Таким образом, группа $G$ действует на $M$, а $H$ является ее стационарной подгруппой. Это означает, что орбита группы $G$ на $M$ накрывается однородным многообразием $G \backslash H$. Следовательно, $H$ замкнута в $G$.

Отметим, что квазиполное многообразие являются наиболее сжатым, т.е. универсально притягивающим объектом в категории всех локально изометричных многообразий. Для любого риманова аналитического многообразия $M^{\prime}$, алгебра векторных полей Киллинга которого не имеет центра, существует локально изометрическое отображение из $M^{\prime}$ в квазиполное многообразие $M$, определенное на всем $M^{\prime}$.

3. Локально однородные многообразия, алгебра векторных полей Киллинга которых имеет нетривиальный центр. Исследуем случай, когда алгебра $\mathfrak{g}$ имеет ненулевой центр $\mathfrak{z}$, и укажем свойства алгебр $\mathfrak{g}, \mathfrak{h}$ и $\mathfrak{z}$, обеспечивающие замкнутость подгруппы $H$ в $G$.

Определим локальную группу локальных изометрий. Рассмотрим произвольное риманово аналитическое многообразие $M$, алгебру Ли $\mathfrak{g}$, состоящую из векторных полей Киллинга на нем, и группу Ли $G$ с алгеброй Ли g. Под локальной группой $G$ (chunk of a group) будем подразумевать малую окрестность единицы группы $G$. Она состоит из локальных изометрий многообразия $M$. Алгебра Ли $\mathfrak{g}$, как правило, не порождает группы изометрий многообразия $M$, но порождает псевдогруппу локальных изометрий. Локальную группу будем обозначать той же буквой, что и группу. Орбита локальной группы локальных изометрий многообразия $M$ является локально однородным многообразием $N$. Заметим также, что локальная группа $H$, порожденная стационарной подалгеброй $\mathfrak{h}$, образует группу изометрий некоторого шара с центром в отмеченной точке многообразия $M$.

Изучим сначала некоторые свойства локальной группы локальных изометрий с точки зрения абстрактных групп преобразований. Рассмотрим локальную группу $G$ как подгруппу группы локальных диффеоморфизмов многообразия $M$ с отмеченной точкой, $G \subset$ Diff $M$. Назовем элемент $\tilde{n} \in G \subset \operatorname{Diff} M$ умножением справа, если существует такой элемент $n \in G$, что для всех $x \in M$, удовлетворяющих условию $x=g(e)$, имеем $\tilde{n}(x)=g n(e)$. Умножение справа на элемент $n$ определено корректно, если для всех $h \in H$ найдется такой $h_{1} \in H$, что для любой локальной изометрии $g \in G$ выполняется равенство $g h n=g n h_{1}$; другими словами, $n$ принадлежит нормализатору $N(H)$ группы $H$ в $G$. Обозначим через $N$ локальную группу, состоящую из элементов $n \in G$, умножение справа на которые в группе $G$ порождают локальные изометрии многообразия $M$, а через $\mathfrak{n}$-ее алгебру Ли. Тогда $h \triangleleft \mathfrak{n} \subset G$. Заметим, что сами умножения справа, т.е. элементы $\tilde{n}$, а также элементы центра $Z$ локальной группы $G$ принадлежат $N$. Обозначим через $M_{0}$ орбиту локальной группы $N$ на $M$. Присоединенное действие элементов $n \in N, g \mapsto n^{-1} g n$, задает локальные изометрии на $M_{0}$.

Найдем подгруппу $G_{0} \subset G$, состоящую из «умножений слева». Рассмотрим отображение $f$ из группы $G$, заданной как группа преобразований множества $G$ в себя, определенное по формуле $f(g)=g(e)=g e$, где $e$ - тождественная локальная изометрия. Тогда, так как $\tilde{n}(e)=e n=n$, то будем считать, что $f(\tilde{n})=n$. На множестве $f(G)$ определим умножение $g_{1} g_{2}=g_{1}(e) g_{2}(e)$. Определенное таким образом умножение превращает $f(G)$ в подгруппу $G_{0} \subset G$. Левые умножения $g \in G_{0}$ дополняются правыми умножениями $\tilde{n}$, т.е. любой элемент $g \in G \subset$ Diff $G$, для которого $g(x)=g x$ при всех $x \in G$, представи́м в виде $g=g_{0} \tilde{n}$, где $g_{0} \tilde{n}(x)=g x n$ при всех $x \in G$. Следовательно, $G=G_{0} N, \mathfrak{g}=\mathfrak{g}_{0}+\mathfrak{n}$.

Теорема 5. Пусть $\mathfrak{g}$ - алгебра Ли всех векторных полей Киллинга на локально однородном римановом аналитическом многообразии $M, \mathfrak{h}$-ее стационарнал подалгебра, $\mathfrak{z}$ - центр алгебpъ $\mathfrak{g}$. Пусть $G-$ односвязная подгруппа, порожденная алгеброй $\mathfrak{g}$, и $H-$ ее подгруппа, порожденная подалгеброй $\mathfrak{h}$. Если $(\mathfrak{p}+\mathfrak{z}) \cap \mathfrak{h}=\mathfrak{p} \cap \mathfrak{h}$, то $H$ замкнута в $G$. 
Доказательство. Предположим противное. Рассмотрим замыкание $\bar{H}$ группы $H$ в $G$ и подалгебру $\overline{\mathfrak{h}} \subset \mathfrak{g}$ подгруппы $\bar{H} \subset G$. Подалгебра $\mathfrak{h}$ является нормальной подалгеброй алгебры $\overline{\mathfrak{h}}$ (см. [8]). Рассмотрим однопараметрическую подгруппу $\bar{h}_{t} \in \bar{H}, \bar{h}_{t} \notin H$, определяемую векторным полем $\bar{X} \in \overline{\mathfrak{h}}, \bar{X} \notin \mathfrak{h}$. Как доказано в [8], в простой компактной подгруппе $P \in G$ существует такой тор $T$, что $H \cap T$ является всюду плотной обмоткой тора $T$. Поэтому можно считать, что $\overline{\mathfrak{h}}_{t} \in T \subset P$. Тогда векторное поле Киллинга $\bar{X}$ касательных векторов к орбитам локальной однопараметрической группы $\bar{h}_{t}$ принадлежит алгебре $\mathfrak{t}$ группы $T$ и, следовательно, $\bar{X} \in \mathfrak{t} \subset \mathfrak{p}$, где $\mathfrak{p}$-алгебра Ли группы $P$. Существует такие окрестность единицы $U$ в группе $G$ и шар $B_{\delta}$ радиуса $\delta$ с центром в отмеченной точке $p \in M$, что все элементы $g \in U$ группы $G$ определяют локальные изометрии из шара $B_{\delta}$ в шар $B_{2 \delta}$ радиуса $2 \delta$ с центром в $p \in M$. Так как элементы $\bar{h}_{t}$ принадлежат замыканию $\bar{H}$ группы $H$ в $G$, то для каждого малого $t$ внутренний автоморфизм $x \mapsto \bar{h}_{t} x \bar{h}^{-1}$ группы $G$ является пределом последовательности внутренних автоморфизмов $x \mapsto h_{n} x h_{n}^{-1}, h_{n} \in H$. Заметим, что любая однопараметрическая подгруппа $h_{t} \in H$ определяет группу изометрий шаpa $B_{\delta}$, так как $h_{t}=\left(h_{t / n}\right)^{n}$, а $h_{t / n} \in U$ и, тем самым, определено на всем шаре $B_{\delta}$ и является его изометрией. Поэтому внутренние автоморфизмы $x \mapsto h_{n} x h_{n}^{-1}$, порождающие те же локальные изометрии, что и умножение на $h_{n}$, определяют изометрии шара $B_{\delta}$. Так как элементы $\bar{h}_{t}$ принадлежат нормализатору группы $H$, то внутренние автоморфизмы $x \mapsto h_{n} x h_{n}^{-1}$ определяют отображения на шаре $B_{\delta}$, являющимися пределами изометрий, то они также задают изометрию шара $B_{\delta}$. Тогда, так как для всех достаточно малых $t$ определена локальная изометрия $x \mapsto \bar{h}_{t} x$, то определена и локальная изометрия $x \mapsto x \bar{h}_{t}$ и, тем самым, локальная однопараметрическая группа изометрий, порожденная умножениями справа на элементы $\bar{h}_{t}$.

Все умножения справа коммутируют с умножениями слева, т.е. с элементами группы $G_{0}$, однако могут не коммутировать друг с другом. Докажем, что локальная изометрия $\bar{h}_{t}$ коммутирует со всеми правыми умножениями. Для этого докажем, что действие элемента $\bar{h}_{t}$ в группе внутренних автоморфизмов группы $G, g \mapsto \bar{h}_{t}^{-1} g \bar{h}_{t}$, задает тождественное отображение на $M_{0}$. Рассмотрим последовательность $h_{n} \in H$, сходящуюся к $\bar{h}_{t}$. Так как $H$ является нормальным делителем в $N$, то $n h_{n}=h_{n} n h_{n}^{\prime}$, где $h_{n}^{\prime} \in H$, то $n H=h_{n}^{-1} n h_{n} H$. Следовательно, внутренние автоморфизмы $g \mapsto h_{n}^{-1} g h_{n}$ индуцируют тождественное отображение на $M_{0}$. Переходя к пределу, получим, что внутренний автоморфизм $g \mapsto \bar{h}_{t}^{-1} g \bar{h}_{t}$ индуцируют тождественное отображение на $M_{0}$.

Так как векторное поле $X$, порождающее локальную однопараметрическую группу $\bar{h}_{t}$, принадлежит компактной подалгебре алгебры $\mathfrak{g}$, то $X$ принадлежит коммутанту $[\mathfrak{g} ; \mathfrak{g}]$ алгебры $\mathfrak{g}$.

Векторное поле $Z$ касательных векторов к орбитам локальной однопараметрической группы $z_{t}$ умножений справа на $\bar{h}_{t}$ является векторным полем Киллинга. Докажем, что $Z$ коммутирует со всеми другими векторными полями Киллинга на $M$, т.е. $Z \in \mathfrak{z}$. Так как для всех $n \in \mathfrak{n}$ элементы $\bar{h}_{t} n$ и $n \bar{h}_{t}$ индуцируют одну и ту же локальную изометрию на $M_{0}$, то $\bar{h}_{t}$ индуцируют тождественное отображение на алгебре $\mathfrak{n}$. Следовательно, векторное поле Киллинга $Z$ (так же, как и $X$ ) принадлежит центру алгебры $\mathfrak{n}$. Для любых элементов $g_{0} \in G_{0}$ и $\tilde{n} \in \tilde{\mathfrak{n}}$, рассматриваемых как автоморфизмы группы $G$, выполняются равенства

$$
g_{0} \tilde{n}(x)=g_{0}(x n)=g_{0} x n, \quad \tilde{n} g_{0}(x)=\left(g_{0} x\right) n=g_{0} x n .
$$

Это означает, что алгебры $\mathfrak{\mathfrak { n }}$ и $\mathfrak{g}_{0}$ коммутируют друг с другом. Следовательно, векторное поле $Z$ коммутирует с алгеброй $\mathfrak{g}_{0}$ и с алгеброй $\mathfrak{g}_{0}+\mathfrak{n}$, т.е. $Z \in \mathfrak{z}$.

Так как $z_{t} \bar{h}_{t}^{-1} H=\bar{h}_{t}^{-1} H \bar{h}_{t}$, то $\operatorname{Exp}(t Z) \operatorname{Exp}(-t X)=z_{t} \bar{h}_{t}^{-1} \in H$. Следовательно, $Z-\bar{X} \in H$. Кроме того, $\bar{X} \in \mathfrak{p}=[\mathfrak{p} ; \mathfrak{p}] \subset[\mathfrak{g} ; \mathfrak{g}]$. Можно доказать, что $Z \notin[\mathfrak{g} ; \mathfrak{g}]$. Тогда $Z-\bar{X} \notin[\mathfrak{g} ; \mathfrak{g}]$, но $Z-\bar{X} \in \mathfrak{h} \cap(\mathfrak{z}+[\mathfrak{g} ; \mathfrak{g}])$. С другой стороны, так как $Z-\bar{X} \in \mathfrak{h}$, а $Z-\bar{X} \notin[\mathfrak{g} ; \mathfrak{g}]$, то $Z-\bar{X} \in \mathfrak{h} \cap(\mathfrak{z}+[\mathfrak{g} ; \mathfrak{g}])$, и $Z-\bar{X} \notin \mathfrak{h} \cap[\mathfrak{g} ; \mathfrak{g}]$. Тогда векторное поле $Z-\bar{X}$ является стационарным, но не принадлежит коммутанту. Следовательно, $\mathfrak{h} \cap(\mathfrak{z}+[\mathfrak{g} ; \mathfrak{g}]) \neq \mathfrak{h} \cap[\mathfrak{g} ; \mathfrak{g}]$. Это доказывает теорему.

Теорема 6. Пусть $\mathfrak{g}$ - алгебра Ли всех векторных полей Киллинга на локально однородном римановом аналитическом многообразии $M, \mathfrak{h}$-ее стационарная подалгебра, $\mathfrak{z}$ - иентр алгебры $\mathfrak{g}, \mathfrak{r}-е е$ радикал. Пусть $G$-односвязнал подгруппа, порожденнал алгеброй $\mathfrak{g}$, u $H$-ее подгруппа, порожденная подалгеброй $\mathfrak{h}$. Если для любой полупростой алгебры $\mathfrak{p} \subset \mathfrak{g}$, удовлетворяющей условию $\mathfrak{p}+\mathfrak{r}=\mathfrak{g}$, имеет место равенство $(\mathfrak{p}+\mathfrak{z}) \cap \mathfrak{h}=\mathfrak{p} \cap \mathfrak{h}$, то $H$ замкнута в $G$. 
Доказательство. Предположим противное и рассмотрим замыкание $\bar{H}$ группы $H$ в $G$. Так же, как и при доказательстве теоремы 5 , рассмотрим однопараметрическую подгруппу $z_{t}$, порожденную умножением справа на элементы однопараметрической группы локальных изометрий $\bar{h}_{t}$ в группе $G$. Пусть $\bar{X}$ - векторное поле Киллинга касательных векторов к орбитам локальной однопараметрической группы локальных изометрий $\bar{h}_{t}^{-1}$, а $Z$ - векторное поле Киллинга локальной однопараметрической группы локальных изометрий $z_{t}$.

Пусть $\mathfrak{p}$ - полупростая подалгебра алгебры $\mathfrak{g}$, содержащая векторное поле $\bar{X}, \bar{X} \in \mathfrak{p} \subset \mathfrak{g}$. Докажем, что $Z+\bar{X} \in \mathfrak{h}$ и $Z+\bar{X} \notin \mathfrak{p}$. В односвязной группе Ли $G$ рассмотрим радикал $R$ (подгруппу, соответствующую подалгебре $\mathfrak{r})$ и полупростую подгруппу $P$, соответствующую подалгебре $\mathfrak{p}$. Тогда $R$ - нормальный делитель группы $G, \mathfrak{r}$ - нормальный делитель алгебры $\mathfrak{g}, R \cap P=e, \mathfrak{r} \cap \mathfrak{p}=0$ и имеет место разложение Леви-Мальцева $G=R P$.

Группа $G$ содержит открытую окрестность единицы, действующую как локальная группа локальных изометрий в окрестности отмеченной точки $p \in M$. Так как $z_{t}$ принадлежит центру группы $G$, то $z_{t} \in R$, и так как подгруппа $H$ является нормальным делителем группы $\bar{H}$ (см. [8]), то $\bar{h}_{t}^{-1} z_{t} H=\bar{h}_{t}^{-1} H \bar{h}_{t}=H$. Следовательно, локальные изометрии $\bar{h}_{t}^{-1} z_{t}$ оставляют точку $p$ неподвижной и поэтому принадлежат стационарной подгруппе $H$. Так как $\bar{X} \in \mathfrak{p}$, а $Z \notin \mathfrak{p}$, то $(Z+\bar{X}) \notin \mathfrak{p}$. Поскольку $(Z+\bar{X}) \in \mathfrak{h}$, то доказанное означает, что для выбранной максимальной полупростой алгебры $\mathfrak{p}$ справедливо соотношение $(\mathfrak{p}+\mathfrak{z}) \cap \mathfrak{h} \neq \mathfrak{p} \cap \mathfrak{h}$, что и доказывает теорему.

В заключение следует заметить, что изложенные результаты могут быть обобщены на другие объекты редуктивной геометрии, прежде всего на псевдоримановы пространства и пространства аффинной связности. Также заслуживает внимания изучение аналитического продолжения произвольных римановых аналитических многообразий.

\section{СПИСОК ЛИТЕРАТУРЫ}

1. Кобаяси Ш., Номидзу К. Основы дифференциальной геометрии. Т. 1. - М.: Наука, 1981.

2. Попов B. A. Аналитическое продолжение локально заданных римановых многообразий// Мат. заметки. - 1984. - 36, № 4. - С. 559-570.

3. Попов В. А. Продолжаемость локальных групп изометрий// Мат. сб. - 1988. - 135 (177), № 1. C. $45-64$.

4. Попов B. А. Аналитическое продолжение локально заданных изометрий псевдориманова многообразия// в кн.: Исследования по математическому анализу, дифференциальным уравнениями и математическому моделированию/ Итоги науки. Юг России. - Юж. мат. ин-т, 2015. - 9. - С. 193-200.

5. Попов В. А. Инфинитеземальные аффинные преобразования и их аналитическое продолжение// в кн.: Исследования по математическому анализу, дифференциальным уравнениями и математическому моделированию/ Итоги науки. Юг России. - Юж. мат. ин-т, 2016. - 10. - С. 195-201.

6. Попов B. А. Алгебра Ли векторных полей Киллинга и ее стационарная подалгебра// в кн.: Исследования по математическому анализу, дифференциальным уравнениями и математическому моделированию/ Итоги науки. Юг России. - Юж. мат. ин-т, 2017. - 11.

7. Хелгасон С. Дифференциальная геометрия и симметрические пространства. - М.: Мир, 1964.

8. Malcev A. I. On the theory of Lie groups in the large// Мат. сб. - 1945. - 16 (38), № 2. - С. 163-190.

9. Mostow G. D. Extensibility of local Lie groups of transformations and groups on surfaces// Ann. Math. 1950. - 52. - P. 606-636.

10. Popov V. A. On a periodic boundary-value problem for the first-order scalar functional differential equation// Differ. Equations. - 2003. - 39, № 3. - P. 310-327.

11. Popov V. A. On closedness of stationary subgroup of affine transformation groups// Lobachevskii J. Math. — 2017. — 38, № 4. - P. 724-729.

Попов Владимир Александрович

Финансовый университет при Правительстве Российской Федерации, Москва

E-mail: vlapopov@gmail.com 